\title{
Toute vérité n'est pas bonne à délibérer
}

Le mot « vérité » est un piège pour la pensée, car il est fortement polysémique. L'on s'en rend d'autant mieux compte pour peu qu'on examine ses usages non en tant que fait, mais en tant que valeur : à quelles conditions « la vérité » est-elle considérée comme une valeur, et quelles significations revêt-elle alors?

\section{Polysémie de la "vérité » : un carrefour axiologique}

Invoquer la vérité, c'est faire référence à une entité valorisée, autrement dit un « bien », ou une valeur au deuxième sens du terme dans la trilogie des trois grands sens du mot «valeur »- une grandeur, un bien, un principe de jugement ${ }^{1}$. Il est même probable qu'elle soit dans la culture occidentale moderne une " valeur fondamentale », au sens où il est peu probable de la rencontrer en tant qu' « antivaleur », c'est-à-dire en tant que principe de dévalorisation. Imagine-t-on quelqu'un déclarer, sans être immédiatement déclaré fou ou provocateur, «Cela n'est pas valable car c'est la vérité ?»

Cela étant posé, le problème commence lorsqu'on tente de spécifier le sens que peut revêtir cette valeur de vérité dans les différents contextes où elle est susceptible d'être invoquée. Nous allons voir que sa grande polysémie fait de la vérité un véritable carrefour axiologique, où se croisent de multiples « registres de valeurs ».

Un premier registre est le registre épistémique, centré sur la valeur de savoir, de connaissance, de « vérité » objective, certifiée par les normes contraignantes de production des données scientifiques. Même si ces normes évoluent au cours du temps et même si ces données se modifient, périmant certains états du savoir établi dans la communauté savante, il n'en reste pas moins que cette relativité effective de la vérité scientifique ne l'empêche pas de fonctionner, à un moment donné du temps, comme le référent partagé par tous - sinon le socle, du moins la visée commune à tous ceux qui se sentent concernés par le registre épistémique de valorisation des objets, des personnes, des actions ou des états du monde. Ainsi « $2+2=4$ » est, sans conteste, une vérité mathématique.

Mais il arrive aussi que l'on parle de "vérité des prix » : là, c'est le registre économique du juste prix qui est sollicité. La « vérité » y renvoie à l'hypothèse qu'un prix (autrement dit la mesure d'une valeur, au premier sens du terme) possède un lien nécessaire avec la nature de l'objet, celle-ci impliquant soit une valeur intrinsèque (hypothèse objectiviste ou substantialiste), soit une valeur socialement construite par le jeu de l'offre et de la demande (hypothèse relativiste ou interactionniste). La « vérité » d'un prix renverrait donc à l'idée qu'il n'existe qu'un seul prix susceptible d'exprimer objectivement la valeur d'un objet quelconque à un moment donné du temps.

\footnotetext{
${ }^{1}$ Cette modélisation a été proposée in Heinich 2017. La suite de cet article s'appuiera sur les propositions développées dans cet ouvrage.
} 
Dans un tout autre domaine, la question de la « vérité » d'un objet de foi a occupé pendant des siècles de larges pans de la théologie et même de la philosophie : « Est-il vrai que Dieu existe?»C'est que la croyance religieuse, comme toutes les croyances, ne se satisfait pas de sa seule existence en tant qu'expérience personnelle, subjective : il lui faut aussi s'assurer que cette croyance, objectivement réelle (elle existe bien en tant que croyance éprouvable et observable), a pour référent un objet tout aussi réel, fût-il situé dans un autre régime d'existence, d'ordre transcendantal. $C^{\prime}$ est à cette condition qu'elle est susceptible d'être partagée, de convaincre et, partant, de devenir une religion. C'est dire que la question de la vérité est essentielle aussi dans ce registre mystique que constitue la famille des valeurs associées à la foi, à la révélation, à la certitude ou, en tout cas, à l'hypothèse de l'existence d'un monde non réductible à l'immanence de l'expérience ordinaire.

La recherche de sens, de signification, est elle aussi soumise à l'exigence de vérité : qu'il s'agisse, trivialement, d'un message crypté ou d'un texte en langue étrangère, ou encore, plus philosophiquement, du sens d'un texte littéraire, d'une œuvre d'art, voire d'un augure, toute interprétation se doit d'être « vraie », c'est-àdire conforme au référent dont elle se propose d'exprimer la signification. Nous sommes là dans le registre herméneutique qui gouverne l'hypothèse que derrière la réalité visible existe un sens non immédiat, qui confère à l'objet en question sa valeur, et qu'il convient de faire apparaître - dans toute sa "vérité », bien sûr.

«Vrai » peut aussi se dire de ce qui est authentique : «Un vrai Rembrandt», « un vrai (ou un véritable) artiste ». On est là dans le registre pur des valeurs associées à l'intégrité du lien entre l'état d'une entité et son origine : le tableau actuel et la main qui l'a peint, le sac de luxe et son prototype tel que sorti de l'entreprise dont il porte la marque, le corps actuel et son état non abîmé par les maladies ou les impuretés, l'intention affichée et l'intention première, etc. Authenticité, santé, sincérité se retrouvent ainsi dans un même souci de pureté, s'agissant d'éviter le faux, la contrefaçon, la duplicité - autrement dit les différentes atteintes à la « vérité » de l'origine.

Un tableau peut également être « vrai » au sens de ressemblant, cette ressemblance fût-elle référée non à l'aspect extérieur du modèle mais à l'intériorité de l'artiste : «Je vous dois la vérité en peinture », proclamait Cézanne. Plus banalement, c'est le réalisme pictural, photographique, cinématographique et, bien sûr, littéraire, qui est concerné par cette exigence de vérité de la représentation. Ainsi la « vérité » d'un tableau de Caravage tient-elle autant à l'illusionnisme des effets picturaux qu'à la trivialité, à l'absence d'idéalisation des modèles. Nous sommes là, bien sûr, dans le registre esthétique, où convergent les valeurs associées à l'exigence de beauté.

Très éloigné du registre esthétique est le registre éthique, associé aux valeurs de moralité, de souci du bien d'autrui, de respect des normes de comportement. Et pourtant, la vérité aussi y a sa part : en effet le mensonge est un tort fait à autrui, dont on trahit la confiance. C'est pourquoi la franchise est une valeur parfaitement morale : «Il faut dire toute la vérité ! » 
« Jurez de dire la vérité, toute la vérité, rien que la vérité » : c'est aussi une valeur dans le registre juridique, qui commande le rapport à la légalité, au respect des règlementations. Et de même que le justiciable appelé à la barre doit la vérité aux magistrats, ceux-ci se mettent au service de la vérité : on sait que l'erreur judiciaire cette atteinte à la vérité de l'imputation des fautes - est la hantise de la Justice, institution vouée à « faire toute la vérité » sur une infraction supposée et, le cas échéant, à la sanctionner en proportion de sa gravité.

Enfin, la vérité est aussi ce que doivent les hommes politiques aux concitoyens qui les élisent, les journalistes aux lecteurs qui leur font confiance. Dissimuler l'origine de ses revenus ou l'existence de conflits d'intérêt est contraire à la déontologie de l'élu, de même que déformer ou masquer les faits l'est à la déontologie du journaliste (qui n'a droit à la dissimulation que pour protéger ses sources, c'est-à-dire les conditions d'établissement de la vérité). C'est que l'information - une information « véridique ", bien sûr - est essentielle au bon fonctionnement d'une démocratie : la vérité relève donc bien aussi du registre civique, celui qui règle le souci de l'intérêt général, du bien commun.

Nous voilà donc face à un complexe carrefour axiologique, où convergent neuf registres parfaitement hétérogènes : épistémique, économique, mystique, herméneutique, pur, esthétique, éthique, juridique, civique. Il n'est guère que les registres aesthésique (le plaisir), domestique (la proximité), fonctionnel (la commodité), ludique (le jeu), réputationnel (le renom, l'honneur) et technique (l'habileté) qui ne fassent pas place à l'exigence de vérité - jusqu'à preuve du contraire...

Voilà qui ne doit pas nous faire douter de l'importance ni de la consistance de cette valeur : quoique rétive au réductionnisme moniste cher à la logique formelle, elle témoigne, par sa polysémie et son hétérogénéité mêmes, de sa force axiologique, en tant qu'elle atteste l'attente d'adéquation entre un énoncé, un chiffre, une émotion, une chose, un acte, un état du monde, et ce dont ils sont présumés tenir leur origine ou leur raison d'être. Incontournable exigence de vérité, donc, non pas en dépit mais en raison même de son caractère axiologiquement hétéroclite.

\section{La seule vérité dont la démocratie a besoin est d'ordre civique}

Arrivés à ce point, que faire de la démocratie au regard de la valeur de vérité?

La réponse va de soi : la démocratie n'a que faire d'une vérité épistémique, économique, mystique, herméneutique, pure, esthétique, éthique ou juridique. La seule vérité dont la démocratie a besoin est d'ordre civique : assurer la transparence de l'information dispensée aux citoyens, l'absence de conflits d'intérêt et, surtout, de corruption des élus, sont des conditions indispensables à la poursuite de l'intérêt général. Le bien commun exige cette vérité-là - et aucune autre.

Il peut sembler naïf de l'affirmer, mais est-il si naïf, aujourd'hui, de rappeler que les règles démocratiques sont exclusives de tout autre régulation, y compris et surtout d'ordre religieux ? Et surtout, est-il si naïf de réaffirmer par les temps qui courent la primauté absolue d'une information vérifiée, produite par des 
professionnels, sur n'importe quel autre énoncé produit par des amateurs sur Internet, non médiatisé par des spécialistes, non soumis à des règles minimales de vérification, pas plus qu'au respect de la différence de nature entre une opinion et un fait ? Les moyens techniques de diffusion des opinions grâce aux réseaux sociaux Facebook, Twitter, YouTube... - ont abouti à une épidémie mondiale de bobards, qui sont probablement pour beaucoup, sinon pour l'essentiel, dans l'emprise croissante des populismes de tous bords.

Dans ces conditions, il est tout sauf superflu de rappeler l'absolue nécessité de respecter la valeur de vérité, contre les propagandistes de « fake news » ou « infox » et les adeptes d'une " post-vérité », auxquels les théories post-modernes, constructivistes et relativistes, ont malencontreusement déroulé un tapis rouge dans le monde académique.

\section{La vérité scientifique n'apporte rien à la démocratie}

Que la seule vérité qui compte en démocratie soit d'ordre civique implique un corollaire, peut-être moins consensuel : c'est que le registre épistémique ne devrait pas avoir sa place dans le débat démocratique. En d'autres termes, la vérité scientifique n'apporte rien à la démocratie.

Certes, l'expertise basée sur les travaux de recherche, et donc soumise aux contraintes de production d'un savoir véridique, est une ressource indispensable pour les décideurs, et il faut en rappeler incessamment la nécessité, les vertus et les exigences - notamment en termes de qualité des experts et de contrôle des conflits d'intérêt, si malmenés notamment en matière d'expertise environnementale. Mais l'expertise n'est qu'une ressource pour les décideurs, c'est-à-dire les responsables administratifs ou les élus, à qui doit finalement revenir - en bon fonctionnement démocratique - la responsabilité des décisions.

En d'autres termes, une décision politique, prise en vue de l'intérêt général, ne doit pas se justifier « au nom de la science », car la science relève de la factualité et non de l'action, encore moins de l'opinion. A chacun ses compétences : le chercheur produit du savoir, l'expert (souvent le même que le chercheur mais dans un contexte d'aide à la décision) applique ce savoir à la résolution d'un problème d'ordre civique, et le décideur s'appuie sur cette expertise pour privilégier - si tout va bien - la meilleure ou la moins mauvaise des options. Mais le décideur n'est pas et n'a pas être un savant - et réciproquement.

A ne pas respecter cette distribution des compétences, on risque les ravages d'un certain scientisme, tel qu'il s'est malheureusement illustré au $X X^{\circ}$ siècle dans le système soviétique : la « dictature du prolétariat » s'appuyant sur un marxisme « scientifique » pour assurer sa prétention à la légitimité politique. Avec les résultats qu'on a connus - et que certains aujourd'hui semblent avoir oublié, lorsqu'ils proclament le droit de la "science sociale », et de la sociologie en particulier, non pas seulement à éclairer les décideurs mais à leur dicter leurs actions. Avec les résultats que l'on connaît aujourd'hui, notamment en matière d'Education nationale. 
Comme l'écrit excellemment Pierre-André Taguieff : «L'idée d'une vérité "définitive" n'appartient pas au savoir scientifique, mais aux dogmes d'une religion révélée. (...) L'histoire des sciences est un vaste système rempli de "vérités définitives". (...) Pas plus que la génétique, l'anthropologie et la sociologie ne sont une morale et une politique. La République a besoin de savants, mais ces derniers ne doivent pas prétendre énoncer des impératifs moraux ni régenter la vie politique. La démocratie n'est rien sans une communauté de citoyens qui discutent librement sur la définition du bien commun, en s'efforçant modestement de s'accorder sur les raisons de leurs désaccords. Aucun savoir scientifique n'apportera le dernier mot sur les questions qui comptent et qui concernent la conduite de la vie. Est-il par ailleurs besoin de rappeler que, dans le domaine du savoir scientifique, aucune vérité n'est définitive ? » (Taguieff $2018: 250$ et 252-253).

\section{La démocratie n'apporte rien à la vérité scientifique}

Mais si une séparation rigoureuse doit être respectée entre le registre civique de la vérité démocratique et le registre épistémique de la vérité scientifique, de sorte que celle-ci ne prétende pas régir celle-là, l'inverse est également vrai : la démocratie n'apporte rien à la vérité scientifique - pour douloureuse que puisse être cette assertion aux yeux de tous ceux pour qui le mot « démocratie » est devenu synonyme de « bien » absolu.

Cela signifie, essentiellement, deux choses. La première est qu'aucune considération relevant des valeurs civiques - égalité, dignité, souci de l'intérêt général, etc. - ne doit interférer dans la production et la transmission des savoirs. Cette proposition peut paraître, là encore, évidente donc superflue, mais imaginons que l'on mette fin aux innombrables et inutiles recherches sur l'innéité de l'intelligence ou des talents, qui ont toutes pour arrière-plan, plus ou moins explicite, la justification ou, au contraire, la délégitimation des inégalités sociales : que d'économies réaliserait-on, qui permettraient d'aider des recherches autrement plus heuristiques!

La deuxième implication est que la démocratie non seulement n'apporte rien à la vérité scientifique, mais lui est foncièrement défavorable. Voilà qui va faire grincer des dents dans les rangs de tous ceux qui n'imaginent pas que deux biens également importants - la démocratie, la science - puissent être, pire qu'indépendants : antagonistes. Et pourtant, c'est bien le cas : dès lors que des considérations autres que le mérite scientifique entrent en jeu dans le traitement octroyé à un chercheur, c'est forcément la qualité de la recherche en tant que telle qui se trouve menacée.

Là encore, cela peut paraître évident, donc inutile à rappeler. Mais réfléchissons à ce que cela signifie concrètement. Première conséquence : il est absurde de réclamer la «parité » hommes/femmes dans les jurys, les recrutements ou les récompenses, car cela va forcément à l'encontre de la sélection par le mérite, en l'occurrence la compétence académique. Tout au plus peut-on veiller à une représentation "équilibrée », voire à un avantage donné aux femmes (ou à tout autre membre d'une catégorie discriminée) en cas de dossiers de qualité équivalente. 
Deuxième conséquence : il est inacceptable que les listes de candidats au « comité national » du CNRS, permettant d'élire les membres des commissions, soient présentées par des syndicats - car quel rapport y a-t-il entre le fait d'être syndiqué et l'expertise nécessaire pour juger de la qualité d'un dossier de recrutement ou de promotion ? Cette anomalie est de notoriété publique, mais aucun responsable n'a eu encore le courage de la corriger, tant est grande l'emprise de la rhétorique « démocratique » dans les institutions scientifiques.

Troisième conséquence : il est contre-productif que la cooptation dans une grande institution universitaire telle que l'EHESS se fasse par une élection faisant intervenir l'ensemble des pairs, car au niveau d'excellence requise, seuls les spécialistes d'un domaine sont en mesure d'évaluer, et donc de décider, au terme $d^{\prime}$ un examen des candidatures et d'une concertation des experts - comme cela se fait d'ailleurs dans toutes les universités du monde entier. Le résultat de cette aberration parfaitement démocratique, mais tout aussi parfaitement anti-scientifique, est bien connu : résultats erratiques, politique de recrutement laissée à l'action en sous-main des réseaux d'amitié ou d'allégeance et, au final, baisse inéluctable de la qualité de l'institution.

Il n'est pas donnéà tout le monde de dire le vrai

C'est que, contrairement aux vœux pieux des idéalistes, le savoir est bel et bien élitiste : il n'est pas donné à tout le monde de dire le vrai. Ce pour quoi, d'ailleurs, il serait urgent d'introduire dans l'Education nationale une formation des élèves aux contraintes d'établissement de la vérité, qu'elle soit d'ordre journalistique ou scientifique (Heinich 2019). Ce qui permettrait, accessoirement, de rappeler aux enseignants que la salle de classe elle-même doit être avant tout le lieu de la transmission des savoirs, plutôt que celui de la discussion ou de l'expression des opinions.

Voilà qui nous ramène à la nécessité de ne pas confondre les différentes arènes de la vérité, toutes légitimes mais pas dans n'importe quel contexte. Or ce qui menace aujourd'hui tant la démocratie que la connaissance, c'est la confusion des arènes civique et scientifique. D'où l'importance de rappeler, contre les vents et les marées des vents dominants de la démagogie, cette vérité, aussi paradoxale puisse-telle paraître : toute vérité n'est pas bonne à délibérer. 


\section{Références bibliographiques}

Heinich, N. (2017) Des valeurs. Une approche sociologique. Paris : Gallimard.

Heinich, N. (2019) « Mon idée pour la France : il faut enseigner les règles présidant à la production de l'information journalistique et du savoir scientifique », LeMonde.fr, 22 février 2019 (https://www.lemonde.fr/idees/article/2019/02/22/mon-idee-pour-la-france-il-fautenseigner-les-regles-presidant-a-la-production-de-l-information-journalistique-et-du-savoirscientifique_5426812_3232.html?xtmc=\&xtcr=1).

Taguieff, P.-A. (2018) «Race» : un mot de trop ? Science, politique et morale. Paris : CNRS éditions. 


\section{Résumé}

Toute vérité n'est pas bonne à délibérer

La polysémie du mot "vérité », du moins en tant qu'il renvoie à une valeur, rend nécessaire la spécification de ses nombreuses acceptions dans différents domaines - scientifique, religieux, éthique, esthétique etc. Appliquées à la question de la vérité en démocratie, ces distinctions permettent d'argumenter en faveur d'une étanchéité entre vérité scientifique et vérité civique : la seule vérité dont la démocratie ait besoin est d'ordre civique (transparence et véracité de l'information), alors que la vérité scientifique ne devrait pas plus la concerner que la démocratie ne devrait régir l'activité scientifique.

\section{Abstract \\ Not all truth is for deliberation}

The polysemy of the word "truth", at least in so far as it refers to a value, makes it necessary to specify its many meanings in different fields - scientific, religious, ethical, aesthetic and so on. Applied to the question of truth in a democracy, these distinctions make it possible to argue in favour of a clear separation between scientific truth and civic truth: the only truth that democracy needs is civic (transparency and veracity of information), whereas scientific truth should not concern it any more than democracy should govern scientific activity.

\section{Notice biographique}

Nathalie HEINICH est sociologue au CNRS (Paris). Outre de nombreux articles, elle a publié près d'une quarantaine d'ouvrages, traduits en quinze langues, portant sur le statut d'artiste et d'auteur (La Gloire de Van Gogh, Du peintre à l'artiste, Le Triple jeu de l'art contemporain, Etre écrivain, L'Elite artiste, De l'artification, Le Paradigme de l'art contemporain) ; les identités en crise (États de femme, L'Épreuve de la grandeur, Mères-filles, Les Ambivalences de l'émancipation féminine, Ce que n'est pas l'identité) ; l'histoire de la sociologie (La Sociologie de Norbert Elias, Ce que l'art fait à la sociologie, La Sociologie de l'art, Pourquoi Bourdieu, Le Bêtisier du sociologue, Dans la pensée de Norbert Elias, La Sociologie à l'épreuve de l'art); et les valeurs (La Fabrique du patrimoine, De la visibilité, Des valeurs). Elle a par ailleurs publié deux récits autobiographiques: Maisons perdues et Une histoire de France.

\section{Biographical note}

Nathalie Heinich, a French sociologist, is senior researcher at the French National Center for Scientific Research (CNRS), and presently works within the Ecole 
des Hautes Etudes en Sciences Sociales (EHESS, Paris). Besides numerous articles in academic and cultural journals, she has published more than thirty books, dealing with : the status of artist (La Gloire de Van Gogh [The Glory of Van Gogh], Du peintre à l'artiste, L'Élite artiste, De l'artification,); the notion of author (L'Épreuve de la grandeur, Etre écrivain); contemporary art (Le Triple jeu de l'art contemporain, L'Art contemporain exposé aux rejets, Le Paradigme de l'art contemporain); the issue of identity (États de femme, Mères-filles, une relation à trois, Les Ambivalences de l'émancipation féminine, Ce que n'est pas l'identité); the history and epistemology of sociology (La Sociologie de Norbert Elias, Ce que l'art fait à la sociologie, La Sociologie de l'art, Pourquoi Bourdieu, Le Bêtisier du sociologue); and, more recently, values (La Fabrique du patrimoine, De la visibilité, Des valeurs). She presented her work through various conferences and lectures in many countries inside and outside Europe. Her books have been translated into fifteen languages. 